\title{
Survol des 140 ans d'histoire des Clercs de Saint-Viateur au Canada et dans la région Joliette-De Lanaudière
}

\section{René Pageau}

Volume 54, 1987

Culture et religion dans la région de Lanaudière

URI : https://id.erudit.org/iderudit/1006963ar

DOI : https://doi.org/10.7202/1006963ar

Aller au sommaire du numéro

Éditeur(s)

Les Éditions Historia Ecclesiæ Catholicæ Canadensis Inc.

ISSN

0318-6172 (imprimé)

1927-7067 (numérique)

Découvrir la revue

Citer cet article

Pageau, R. (1987). Survol des 140 ans d'histoire des Clercs de Saint-Viateur au Canada et dans la région Joliette-De Lanaudière. Sessions d'étude - Société canadienne d'histoire de l'Église catholique, 54, 69-81.

https://doi.org/10.7202/1006963ar

Tous droits réservés ㄷ Les Éditions Historia Ecclesiæ Catholicæ Canadensis Inc., 1987
Ce document est protégé par la loi sur le droit d'auteur. L'utilisation des services d’Érudit (y compris la reproduction) est assujettie à sa politique d'utilisation que vous pouvez consulter en ligne.

https://apropos.erudit.org/fr/usagers/politique-dutilisation/ 


\title{
Survol \\ des 140 ans d'histoire des Clercs de Saint-Viateur au Canada et dans la région Joliette-De Lanaudière
}

\author{
René PAGEAU \\ Supérieur provincial de Joliette \\ et président des Clercs de Saint-Viateur canadiens
}

C'est presque une épopée où la légende se mêle à l'histoire que ces 140 ans de présence viatorienne au Canada! Je vous entretiendrai d'abord du tout début de la fondation de Querbes en France, de son implantation au Canada et dans les autres pays à partir du Canada, mais surtout de l'influence des Clercs de Saint-Viateur dans la région Joliette-De Lanaudière et aussi de l'influence de certaines personnalités natives de la région qui ont marqué la congrégation.

L'aventure a commencé alors que $M^{g r}$ Ignace Bourget venait d'être nommé, en 1840, évêque du diocèse de Montréal qui comprenait L'Industrie. Sa préoccupation majeure était l'éducation et l'instruction des jeunes. Il multipliait les invitations auprès des communautés religieuses d'éducateurs pour venir travailler dans son diocèse. À cette époque, plusieurs communautés religieuses venaient de voir le jour en France et s'empressèrent de répondre affirmativement à son invitation. $\mathrm{M}^{\mathrm{gr}}$ Bourget inspira de plus plusieurs fondations de communautés religieuses à Montréal.

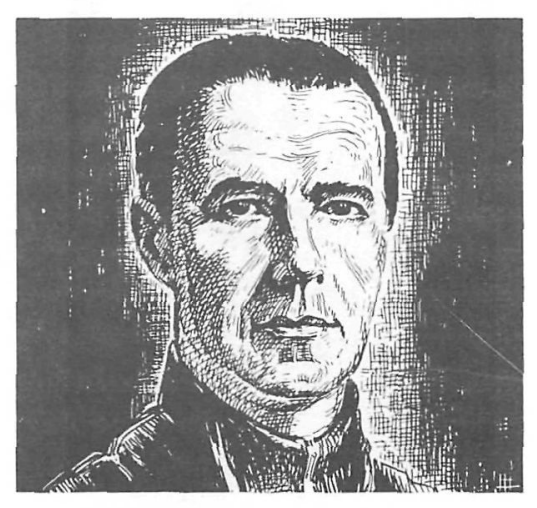


La communauté des Clercs de Saint-Viateur était toute jeune. Le P. Querbes, son fondateur, en avait eu l'idée vers 1828, alors qu'il était curé de Vourles, petit village tout près de Lyon, en France. On était au lendemain de la Révolution française et les jeunes étaient laissés à eux-mêmes. Le P. Querbes voulait former une association de maîtres d'école, de chantres et de sacristains; il voulait d'abord rassembler des personnes qui seraient chargées à la fois d'enseigner la doctrine chrétienne aux enfants et de seconder les prêtres dans les petites paroisses. Cette association serait au service des autels et de l'éducation chrétienne de la jeunesse.

Pour diverses raisons, dans le contexte de l'Église du temps, cette association se transforma en congrégation religieuse qui porta le nom de Clercs paroissiaux ou Catéchistes de Saint-Viateur. Elle fut fondée en novembre 1831, puis approuvée par Rome, le 21 septembre 1838 par une décision de la Congrégation des évêques et réguliers et le décret fut signé par le cardinal Sala, le 27 septembre de la même année.

Un an après sa nomination au siège épiscopal de Montréal, Mrr Bourget, en revenant de sa visite à Rome, fit un arrêt à Lyon. C'était entre le 10 et le 13 août 1841. Il fit demander au P. Querbes de venir le rencontrer, mais on ne sait pas si la rencontre eut lieu. Trois ans plus tard, en février 1844, puis au début de septembre, son vicaire général, $M^{g r}$ Hudon, rencontre le P. Querbes qui lui remet pour son évêque le «petit volume des Règles et constitutions» de la jeune communauté. La correspondance entre $\mathrm{M}^{\mathrm{gr}}$ Hudon et le P. Querbes montre que celui-ci avait accepté la fondation au Canada avant le 31 juillet 1845. En décembre 1846, $M^{\text {gr }}$ Bourget se rend à Lyon, et de là, le 4 décembre, à Vourles, où le $\mathrm{P}$. Querbes lui présente les novices. Puis en 1847 , entre le 5 et le 10 mars, $M^{\text {gr }}$ Bourget rencontre à Vourles une seconde fois, le Père Querbes.

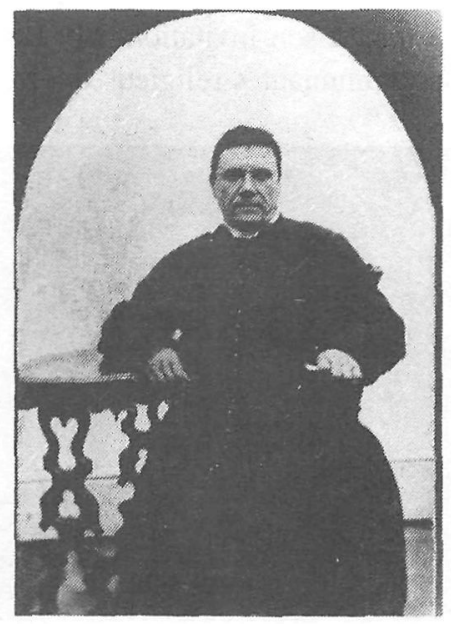


Le fondateur présente la communauté de Vourles à $\mathrm{M}^{\mathrm{g}}$ Bourget, composée d'une vingtaine de religieux et de novices vivant à Vourles et dans les environs. On raconte que $\mathrm{M}^{\mathrm{g}}$ Bourget avait demandé à brûle-pourpoint aux confrères: «Qui veut venir travailler au Canada?» Tous se lèvent pour manifester leur désir de répondre à l'invitation de l'évêque. Un seul demeure assis. Il a 38 ans. Il est trapu, entêté, taciturne. Lui ne voulait pas traverser les mers et venir au Canada. Mais l'évêque de Montréal se fait plus insistant et l'interpelle personnellement. C'était le F. Étienne Champagneur. Il y est venu, avec deux autres frères, Fayard et Chrétien. Il est devenu prêtre malgré lui, parce que l'enseignement au Canada était cléricalisé et que le fait d'être prêtre permettait d'ouvrir des portes sur l'avenir à la jeune communauté.

Quelques semaines plus tard, les trois religieux s'embarquent pour le long voyage en bateau, accompagnés par $\mathrm{M}^{\mathrm{gr}}$ Bourget qui leur donne des cours d'anglais durant la traversée. Ils arrivent à Montréal le 27 mai 1847 et dans la nuit, toujours en bateau, ils remontent le fleuve, de Montréal à Lavaltrie. C'est le 28 mai à 8 heures qu'on vient les chercher en diligence pour les conduire à L'Industrie, là-même où se trouve actuellement la Maison provinciale de la Communauté.

À peine arrivés à L'Industrie, les Clercs de Saint-Viateur prennent la direction du Collège Joliette, fondé un an plus tôt et qui deviendra au fil de ses 140 ans d'histoire, le Cégep Joliette-De Lanaudière.

De Joliette, ils essaimèrent aux États-Unis, en Chine continentale, à Taïwan, au Japon, en Afrique, au Pérou et en Haïti, en passant par Montréal, l'Abitibi, le Saint-Laurent, le Lac Saint-Jean, la Côte-Nord et le Manitoba. Chacune de ces fondations est à elle seule une épopée où, au nom du Christ, on défiait presque le bon sens. Les confrères qui sont allés fonder en Abitibi peuvent encore vous parler longuement de leurs premières années d'enseignement en 1935 dans des poulaillers convertis en petites écoles de villages.

En 1903, sous la loi Combes, les religieux sont chassés de la France. Trente-et-un confrères français passent au Canada les 24 février, 7 juin, 27 août et viennent, par la force des choses, prêter main-forte à leurs confrères canadiens. Au cours des ans, des Canadiens sont même retoumés en France pour aider leurs confrères français dont le recrutement était plutôt lent.

À l'ouverture de la mission du Canada, à Joliette, la jeune communauté ne comptait pas tout à fait dans l'ensemble 120 religieux. Et les jeunes Canadiens, frères et pères, ne tardèrent pas à venir grossir les rangs de la toute première équipe venue de France. Quelques ecclésiastiques de grande stature, intéressés au projet viatorien, se sont joints à la communauté en de- 
venant Clercs de Saint-Viateur: le P. Pascal Lajoie a été 29 ans, supérieur général (1890-1919) et le P. Cyrille Beaudry, supérieur du Séminaire durant 36 ans (1864-1867, 1871-1882, 1883-1904).

À la mort du P. Querbes survenue le $1^{\text {er }}$ septembre 1859 , il fallut déléguer un représentant de la branche canadienne pour l'élection d'un successeur. C'est le P. Pascal Lajoie qui fut délégué. Il était le premier Clerc de Saint-Viateur canadien. Il fut curé de l'église Saint-Charles-Borromée (la cathédrale actuelle) de 1864 à 1880 . Nommé supérieur provincial de la mission canadienne, comme successeur du P. Champagneur, de 1870 à 1880 , il exerça ces fonctions tout en gardant son poste de curé. Il fut ensuite vicaire de la congrégation pendant dix ans et supérieur général de la communauté de 1890 à 1919. Le P. Michel Roberge, de Saint-Cuthbert, fut aussi supérieur général de 1923 à 1937. Et le P. Paul-Émile Farley, de Saint-Gabriel, fut supérieur général de la Congrégation, de 1937 à 1946. On se souvient encore du P. Joseph Latour, de Sainte-Élisabeth, qui a été à la tête de toute la Communauté canadienne de 1929 à 1938.

Ce qui fait la force d'une communauté, c'est qu'elle est une institution. Une institution nourrie du dynamisme des bonnes volontés qui mettent en commun leur avoir et leur être en vue d'une œuvre à accomplir. On méprise parfois l'institution, mais c'est parce qu'un organisme est institutionnalisé qu'il traverse courageusement les tempêtes sociales de toutes sortes et qu'il peut faire œuvre durable. Mettre au service d'une mission à accomplir les talents, les compétences et l'immense dévouement de chacun dans l'harmonie des différences, voilà la force des communautés religieuses. Et cette force de la mission suscite le bénévolat et la générosité. De plus, l'exigence de la voie des conseils évangéliques et le désir de réaliser la volonté de Dieu font partie de la mystique des dévoués, des consacrés. Tous les religieux, du plus humble au plus célèbre, étaient au service des mêmes œuvres apostoliques. L'idéal de la sainteté faisait pousser loin la fidélité dans le dévouement et l'anonymat. Derrière les grandes personnalités qui ont influencé le milieu et dont le rayonnement se fait sentir encore aujourd'hui, beaucoup de mains généreuses, bénévoles et fidèles servaient dans l'ombre l'œuvre d'envergure qui faisait émerger la personne inspiratrice du projet.

Il ne faut pas oublier aussi qu'une épopée ne s'écrit pas en vase clos. Nous avons été soutenus, aidés, encouragés par nos anciens élèves, les parents de nos élèves, les prêtres du milieu et l'épiscopat qui croyait en notre option de vie et en nos initiatives, et qui nous ont permis d'incarner la mission viatorienne partout où l'Église nous envoyait. Une très grande amitié s'est développée entre la communauté et $\mathrm{M}$. et Mme Barthélemy Joliette, entre la communauté et certains évêques dont $\mathrm{M}^{\mathrm{gr}}$ Bourget et $\mathrm{M}^{\mathrm{gr}}$ Papineau, 
évêque de Joliette du 24 août 1928 au 29 février 1968. Ces liens d'amitié ont favorisé l'éclatement des œuvres des Clercs de Saint-Viateur. M ${ }^{\text {gr }}$ Papineau tenait à ce que toutes les écoles de garçons de Joliette soient dirigées par les Clercs de Saint-Viateur. Il fallait faire la sourde oreille devant les demandes des autres évêques pour répondre à l'exigeante amitié qui nous liait à $\mathrm{M}^{\mathrm{gr}}$ Papineau.

À l'époque, les représentants de la Commission scolaire signaient des contrats avec les représentants de la communauté pour avoir un certain nombre de religieux par école. On était loin des contrats individuels et personnels. C'était le responsable de la communauté qui devait trouver un professeur si, à la dernière minute, on ouvrait une classe de plus ou si un religieux était malade. C'est le cas de le dire, tous les religieux, pères et frères, se consacrèrent à l'œuvre de l'enseignement du matin au soir, qu'ils en aient eu les aptitudes ou non!

Les Clercs de Saint-Viateur ont enseigné dans toutes les écoles élémentaires de la ville de Joliette, tout en ayant la responsabilité des enfants de chœur, de l'organisation des loisirs, de la direction des manécanteries; plusieurs avaient le privilège d'être le chauffeur du curé. Ils assurèrent aussi la direction de petites écoles de villages:

$\begin{array}{ll}\text { à Sainte-Élisabeth } & (1848-1849) \\ \text { à Berthierville } & (1848-1969) \\ \text { à Saint-Roch } & (1857-1894) \\ \text { à Saint-Jacques } & (1854-1855) \&(1860-1871) \\ \text { à Rawdon } & (1866-1878) \&(1934-1959) \\ \text { à Lanoraie } & (1874-1915) \&(1919-1961) \\ \text { à Saint-Barthélemy } & (1884-1937) \&(1950-1961) \\ \text { à l'Épiphanie } & (1915-1970) \\ \text { à Crabtree } & (1937-1959) \\ \text { à Saint-Félix } & (1945-1961) \\ \text { à Saint-Côme } & (1866-1867) \&(1948-1955)\end{array}$

On raconte que, dans un village de la région, un président de la Commission scolaire avait dit à une réunion des commissaires: «Il nous faut des petits frères pas chers comme à Lanoraie».

Aussitôt qu'ils purent établir le cours secondaire, jusqu'à la douzième année dans ces milieux, les Clercs de Saint-Viateur le firent aussi avec une généreuse audace, d'abord à l'Académie Saint-Viateur de Joliette devenue école supérieure, ensuite à Berthierville, puis à l'Épiphanie. Dans toutes 
ces écoles publiques, ils recrutèrent des jeunes pour le séminaire de Joliette, le collège Saint-Joseph et le juvénat de Berthierville, et l'orphelinat Saint-Georges de Joliette.

Plusieurs Franco-Américains vinrent faire leurs études au séminaire de Joliette. La communauté fonda un «high school» à Berthierville et un à Rawdon pour répondre aux besoins des Franco-Américains qui étaient de plus en plus nombreux chaque année. Ces institutions avaient acquis une telle réputation aux États-Unis, qu'on demanda aux Clercs de Saint-Viateur d'ouvrir, à Malboro, un «high school» qui fut sacrifié, après quelques années, pour renflouer les écoles du Québec. Parmi les confrères américains, il y avait de fort bons professeurs d'anglais. Ceux qui travaillent encore au Québec n'ont jamais pardonné à la communauté la fermeture de cette école américaine. Plusieurs Franco-Américains étaient devenus Clercs de SaintViateur avec l'espoir de retourner enseigner dans leur pays. «On le leur avait promis», nous disaient-ils!

Au moment de la Révolution tranquille en 1960, les Clercs de SaintViateur du Canada comptaient plus de 1100 religieux répartis en quatre provinces religieuses. Ils dirigeaient cinq collèges classiques dont l'un en Ontario et de nombreuses écoles supérieures, du Manitoba au Québec, de la lointaine Abitibi à la Côte-Nord. Plusieurs écoles d'agriculture étaient aussi animées par des religieux devenus agronomes.

À travers toute l'histoire de ces maisons d'enseignement, il est facile de mesurer l'ampleur de l'influence des Clercs de Saint-Viateur dans la région De Lanaudière. Une famille religieuse a une mentalité, une méthode d'enseignement, un esprit, une tradition qui font partie de son héritage. Les archives de la Maison provinciale disent clairement comment l'implantation et l'enracinement des Clercs de Saint-Viateur au Canada ont été intimement mêlés à la naissance et à l'histoire de la ville de Joliette depuis 1847.

Grâce à l'aide et au soutien de l'ensemble, le charisme de quelquesuns rayonne, rassemble des disciples, crée une mentalité qui influence des générations de confrères et des générations d'étudiants. Et quand cette personnalité rayonne également à l'extérieur de son collège, et qu'elle rejoint le public de toute une région, et qu' un de ses disciples continue son œuvre, et qu'un autre prolonge encore l'influence de celui qui a donné naissance à cette œuvre, et que cette œuvre continue à inspirer un milieu, et cela, pendant 25 ans, 50 ans, 100 ans, et que la communauté appuie ceux qui y travaillent, c'est une tradition qui s'édifie et invite au respect et à la fidélité.

Pour bien vous faire comprendre ma pensée, faisons une brève tournée de la région. Commençons par Saint-Norbert. Un coup d'œil sur l'église qui est de l'architecte, le P. Joseph Michaud. Puis tout près, passons 
par la paroisse de Saint-Viateur qui honore le patron de la communauté, et filons vers Berthierville. Un des fondateurs, le F. Fayard, est demeuré ici au collège où les Clercs de Saint-Viateur ont enseigné près de 140 ans. Empruntons la rue Saint-Viateur. Je rappelle aussi qu'au collège de Berthier a vécu le F. Antoine Bernard, grand historien de l'Académie et de la Louisiane, historien de notre communauté, et des communautés des Sœurs de Saint-Paul de Chartres et des Sœurs Hospitalières de Saint-Joseph.

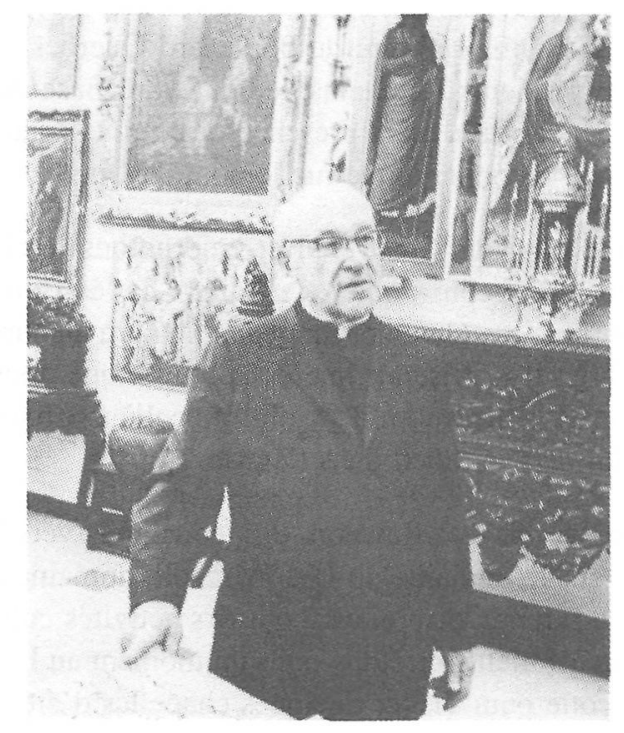

Après un bref arrêt à l'église de Saint-Thomas dont le P. Michaud est aussi l'architecte, entrons à Joliette par le boulevard Père-Wilfrid-Corbeil. À gauche, vous avez le musée dont le P. Corbeil a été le principal artisan. On rappelle son souvenir à l'entrée et une plaque rappelle aussi l'importante contribution des Clercs de Saint-Viateur qui y ont déposé leur collection d'œuvres d'art.

Passons devant l'ancien Séminaire devenu Cégep Joliette-De Lanaudière, saluons le monument du P. Cyrille Beaudry avant d'entrer visiter la salle académique Rolland-Brunelle et la galerie d'art Maximilien-Boucher. Puis passons devant la cathédrale et remarquez que les deux grandes salles portent le nom de deux frères qui ont été sacristains durant de très longues années: Carter et Tétreault. Ensuite, il y a le Centre Champagneur et le noviciat Saint-Viateur dont le P. Wilfrid Corbeil fut l'architecte. Montons au premier étage visiter la chapelle avec ses fameuses verrières et ses nombreuses sculptures. En sortant de la maison provinciale des Clercs de SaintViateur, prenons la rue Saint-Louis nommée en 1'honneur de Louis 
Querbes, fondateur de la communauté; revenons ensuite par la rue SaintViateur. Allons maintenant sur la rue Lajoie. Un peu plus loin, nous avons aussi le parc Lajoie et l'école Lajoie.

Qui n'a pas connu l'Académie Saint-Viateur devenue l'École supérieure Saint-Viateur qui abrite aujourd'hui des services communautaires? Regardez là-haut et vous verrez la statue du patron de la communauté. Il y eut aussi la maison Querbes qui rappelait la mémoire du fondateur et l'écơle Fayard dans Sainte-Thérèse, détruite par ies fiammes, qui rappelait la mémoire du F. Fayard. Et que dire du boulevard Querbes qui est devenu, au grand regret de plusieurs Joliettains, le boulevard Base-de-Roc? À l'occasion du $140^{\circ}$ anniversaire de la présence des Saints-Viateurs à Joliette, serait-il opportun de redonner à une rue le nom de Querbes?

Filons maintenant vers Saint-Paul et empruntons le chemin CyrilleBeaudry. Ensuite, engageons-nous vers Saint-Charles-Borromée: l'école Lorenzo-Gauthier et la rue Gauthier rappellent le nom du curé fondateur de la paroisse du Christ-Roi. Il serait intéressant aussi que nous empruntions la rue Père-Michaud. À Rawdon, il y aurait le collège Champagneur à visiter et les murales du P. Corbeil dans l'église.

Après une brève visite à Rawdon, dirigeons-nous vers le camp musical qui se trouve sur le domaine du Lac Priscault, domaine que les Clercs de Saint-Viateur ont rendu disponible pour des activités culturelles et pastorales. En entrant à Joliette, arrêtons-nous un moment au Lac Noir, au Lac Vert et au Lac Ayotte pour visiter quelques chapelles d'été dont le P. Wilfrid Corbeil fut l'architecte. En passant près de l'Auberge de Joliette, empruntons la rue Livernoche qui rappelle le P. Jean Livernoche et bien d'autres confrères qui ont fait beaucoup pour les sports dans les écoles du milieu.

La région est sillonnée d'œuvres de l'architecte Michaud, du peintre et de l'architecte Corbeil et du sculpteur Boucher. Tous ces noms appartiennent à la grande famille de la congrégation des Clercs de Saint-Viateur qui ont marqué la région.

Je ne voudrais pas retenir l'attention uniquement autour de l'œuvre du séminaire étant donné que les Clercs de Saint-Viateur ont aussi travaillé dans des œuvres spécialisées comme au tout début à l'école industrielle où l'on enseignait les métiers, à l'orphelinat Saint-Georges pour les jeunes sans familles, à la maison Querbes pour les retraites fermées et à l'école d'Agriculture de Saint-Barthélemy. Que dire aussi de l'école de haut-savoir où l'enseignement théologique était donné à tous les Clercs de SaintViateur qui optaient pour le sacerdoce? Nous avions là une bibliothèque en spiritualité et en théologie qui faisait l'envie de plusieurs grandes institu- 
tions. Cette maison est maintenant devenue le Centre de réflexion chrétienne.

Malgré tout, force nous est de reconnaître que, dans chaque région, le collège classique était un foyer rayonnant de culture. Dès 1914, A.-C. Dugas, dans Gerbes de souvenirs, nous renseigne sur l'atmosphère culturelle dans laquelle baignait toute la vie du séminaire. Dès 1927, le P. Joseph Charlebois, alors supérieur au Séminaire, publiait Les Anciens du Séminaire: écrivains et artistes.

Depuis toujours, le Séminaire de Joliette a été reconnu dans tout le Québec comme étant un foyer de culture grâce à la communauté des Clercs de Saint-Viateur, ouverte à l'expression artistique sous toutes ses formes. Musicien, le F. Vadeboncoeur est à l'origine de cette tradition. Il est entré en communauté avec le P. Pascal Lajoie. Ils furent les deux premiers Canadiens à devenir Clercs de Saint-Viateur.

Dès le début, une attention particulière fut donnée au chant et à la musique sacrés. Cette réputation attirait les jeunes qui avaient un certain talent musical. Après le F. Vadeboncoeur, il y eut les frères Georges et Louis-Camille Paul, le P. Charlebois, le F. Larivière, le P. Bellemare, le P. Brunelle, le P. Marion, le P. Lindsay, et d'autres encore, sans compter le peintre Wilfrid Corbeil et l'écrivain Gustave Lamarche qui étaient de fort bons musiciens. Cette longue tradition a donné aussi aujourd'hui le festival d'Été de Lanaudière, le camp musical, l'école de musique et a fait de la ville $J_{O}$ liette, sol de musique.

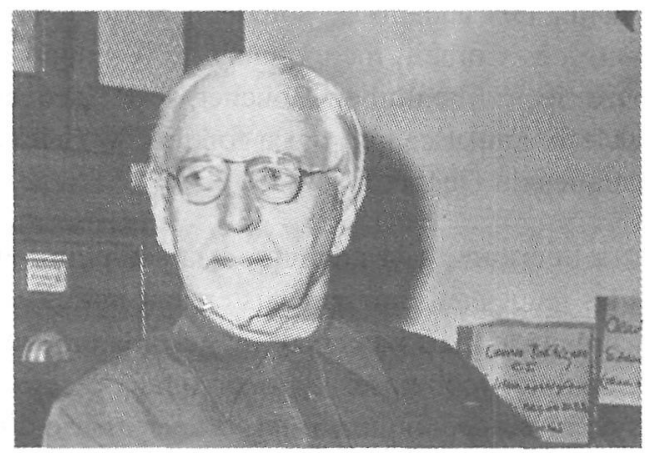

Les Clercs de Saint-Viateur ayant toujours favorisé les écrivains, il y aurait un long chapitre à écrire sur les manuels scolaires mais je ne retiens que l'Histoire du Canada des pères Paul-Émile Farley et Gustave Lamarche, écrite pour le cours supérieur, publiée en 1934 et qui, en 1968, a été reprise par l'équipe du Boréal Express; le manuel d'Histoire du Canada pour le cours primaire, œuvre du F. Alphonse Grypinich; les manuels de sciences naturelles écrits par le F. Léo Brassard et illustrés par le P. Maxi- 
milien Boucher; les cahiers de chimie du P. Réal Aubin. Mais il y eut aussi les fameux Carnets viatoriens, revue trimestrielle et d'intérêt général, fondée et dirigée par le P. Gustave Lamarche de 1936 à 1955. La revue $L e$ Jeune naturaliste, fondée en 1950 par les Clercs de Saint-Viateur, dont le F. Léo Brassard était la cheville ouvrière, est devenue Le Jeune scientifique en 1962 et Québec science en 1969.

Le climat communautaire a permis à plusieurs de naître à l'écriture. Ces écrivains s'étaient fait la main dans $L^{\prime}$ Estudiant, joumal du séminaire, et dans les différentes revues de la communauté. Ils y étaient soutenus et encouragés par des maitres de première qualité qui étaient des éducateurs, des initiateurs, et qui inspireront des générations d'étudiants de confrère. Je pense ici aux de Grandpré, Lefebvre, Pinard, Lamarche, Farley.

Un mot sur la prodigieuse activité de l'animateur culturel et artistique que fut le fameux Corbeil. Dès 1931, il fonde au séminaire un studio d'art qui sera fréquenté par des générations et des générations d'élèves dont plusieurs parmi eux sont devenus Clercs de Saint-Viateur. Il nous réserve annuellement une exposition de ses œuvres. Il prépare des expositions d'art sacré. Il prend la parole hautement pour dénoncer le mauvais goût dans le réaménagement des églises ou pour encourager ce qu'il estime être de bon goût. Il fonde le Rétable qui regroupe des artistes et des artisans de qualité. Il préside au réaménagement de plusieurs églises dans la région. Ces décors de théâtre ne manquent pas d'inspiration et suscitent la curiosité. On vient de Montréal assister aux représentations des pièces jouées par les élèves du séminaire. Les anciens se souviennent de Jonathas du P. Lamarche. À la suite des Corbeil, mentionnons le F. Edgar Plante, peintre, graveur et orfèvre, le P. Maximilien Boucher, le P. Jacques Houle, le F. Bruno Hébert et le F. Paul Beaupré qui a fondé Vision, revue des professeurs d'arts plastiques du Québec.

L'initiation à l'écriture, à la peinture, à la sculpture, au théâtre, à la musique attirait les jeunes au séminaire. Ils étaient initiés non seulement à partir des grands maîtres, mais ils étaient invités à la création. Ils devaient écrire, peindre, sculpter, composer et jouer quelques pièces de théâtre, quelques pièces de musique, comme le faisait leur maître d'école.

Les sciences ne faisaient pourtant pas figures de parents pauvres à côté des lettres et des arts. Les sciences physiques et naturelles ont toujours trouvé une grande place aussi dans nos maisons d'éducation. Un filon, au hasard des années, pourrait être suivi quand je pense au premier traité de botanique attribué au F. Augustin Fayard, édité à 500 exemplaires en 1848, et quand je lis les chroniques de nos maisons d'éducation. Les anciens du séminaire, qui ont actuellement plus de 60 ans, se souviennent du P. Joseph 
Morin, devenu le premier doyen de la Faculté des sciences à l'Université de Montréal, et du P. Josaphat Asselin, éminent professeur de chimie, enseignant en même temps à la même université et au séminaire.

Plusieurs ont reçu leur formation au camp des Jeunes Explos fondé et dirigé par le $\mathrm{F}$. Léo Brassard, il y a plus de 35 ans. Il a inspiré nombre de Clubs de jeunes naturalistes dans la région et à l'extérieur. Son école d'été a regroupé plusieurs confrères qui, avec de nombreux autres collaborateurs, ont fondé d'autres camps de sciences naturelles dans la région de $\mathrm{La}$ Malbaie où des générations de scientifiques se sont levées. Il y avait eu les Ouellette et les Robert de Montréal dont les collections d'insectes ont été léguées à l'Université de Montréal. Il y eut aussi les Mongeau, les Genest et les Larochelle. Signalons que le F. Jean-René Mongeau, biologiste, travaille, depuis de nombreuses années, au Service de l'aménagement de la faune au ministère du Tourisme, de la Chasse et de la Pêche. Il s'est fait remarquer par ses nombreuses publications scientifiques, comme le F. Adrien Robert d'ailleurs. Ces confrères spécialistes ne sont certes pas étrangers aux recherches qui se font depuis longtemps aux Jeunes Explos.

Mentionnons encore une fois l'œuvre magnifique de quelques confrères de Montréal qui ont eu une grand influence dans l'orientation de l'enseignement secondaire au Québec et qui ont du même coup influencé notre milieu. Aux alentours de 1922, il revient au F. Wilfrid Coderre, natif de Joliette, et au F. Joseph Piédalue de travailler à promouvoir la naissance des écoles secondaires et supérieures. Les volumes du P. Philippe Deschamps, professeur à l'Université Laval, ont aussi guidé des générations d'étudiants dans l'art d'écrire. La revue de cinéma Séquences, fondée par le $\mathrm{F}$. Léo Bonneville il y a plus de 25 ans, a inspiré les ciné-clubs de toutes les institutions dirigées par les Clercs de Saint-Viateur.

Si les Clercs de Saint-Viateur sont fiers du passé, ils n'en sont pas les adorateurs. Le temps les tire en avant. Et c'est dans une vision d'avenir qu'ils ont accepté d'ouvrir des missions, à la demande des papes, en Amérique latine et en Extrême-Orient. Là encore, ils forcent les événements pour que les écoles soient prises en main par les gens du milieu. Lorsqu'une école, comme le séminaire qui devient l'académie Antoine-Manseau, continue une œuvre d'éducation de qualité, lorsqu'au collège Champagneur les principaux postes sont entre les mains de collègues laïques, lorsque l'immense collège de Taïwan est cédé à une communauté autochtone, lorsque plusieurs postes de commande du collège Rakusei de Kyoto sont confiés à des laïques, la communauté croit réussir à accomplir ce que l'Église lui demande. Elle sert toujours la cause de l'éducation chrétienne d'une façon parfois différente et dans un autre contexte social. 
C'est à grands traits que j'ai tenté de vous tracer l'histoire d'une communauté d'éducateurs. J'aurais aimé vous parler aussi de tous les collaborateurs, de tous les disciples des grands animateurs culturels dont je vous ai entretenus et ils sont multiples. J'aurais aimé vous dire aussi comment la communauté s'est ressaisie au cœur de la Révolution tranquille. Y a-t-il des organismes sociaux qui ont été plus remis en question que les communautés religieuses depuis 1960? J'aurais aimé vous dire comment les Clercs de Saint-Viateur sont sortis vivifiés de ces temps difficiles, comment ils ont traversé ce désert avec la certitude que la communaauitié étãit voulue par Dieu plus qu'elle ne le voulait elle-même. Ces temps difficiles l'ont rendue plus démocratique dans le choix de ses orientations, plus fraternelle dans sa vie quotidienne, plus libre dans ses façons de servir.

Mon propos est forcément injuste. Il est trop rapide et sans nuances. J'en ai oublié plusieurs. On me le pardonnera. Quand une œuvre d'éducation s'élabore pendant 140 ans, nous nous apercevons que les chemins sont nombreux et diversifiés, mais que la mission des Saints-Viateurs est unique. Partout où ils ont passé, partout où ils passent, ils font œuvre d'éducation, même en paroisse. Et à travers tous les talents, tous les moyens mis à leur disposition, toutes les collaborations, c'est de Dieu qu'ils essaient de témoigner.

Notre Constitution traduit notre mission en ces termes: «Annoncer Jésus-Christ et son Évangile, et susciter des communautés où la foi est vécue, approfondie et célébrée.» Cette mission, nous la réalisons à travers notre travail d'éducateurs et nos tâches professionnelles et pastorales, en nous efforçant d'intégrer les valeurs de la foi à la culture de notre temps $\left(\mathrm{n}^{\circ} 8\right)$.

Au début, notre communauté portait le nom de Clercs paroissiaux ou Catéchistes de Saint-Viateur. Le travail en paroisse fait donc partie de notre mission d'éducateurs. Le P. Lorenzo Gauthier a fait sa marque dans le milieu comme prédicateur et écrivain. On se rappelle les mercredis de Saint-Joseph qui attiraient des gens de tous les coins du diocèse. Il a fait œuvre de catéchiste par ses publications. Il est le curé fondateur de la paroisse du Christ-Roi. Dans cette veine, mentionnons le F. Armand Billette qui, en 1947, a publié un catéchisme pour les jeunes de première et de deuxième années. Viens vers le Père a été tiré à 500,000 exemplaires.

Le P. Norbert Fournier a été, de l'avis de $M^{\text {gr }}$ Coderre, l'un des principaux artisans et pionniers du renouveau catéchétique au Canada français. Il est l'auteur du premier ouvrage sur le renouveau catéchétique canadien: Exigences actuelles de la catéchèse (1960). Il a été directeur fondateur d'un département de catéchèse à l'Université de Montréal en 1960.

Soulignons en outre deux autres confrères de Montréal: le F. Philippe Champagne, originaire de Lanoraie, qui a fondé il y a plus de 25 ans l'As- 
sociation des Comités de liturgie engagés (ACLE) et qui s'est donné comme objectif de former des animateurs en liturgie pour animer les communautés chrétiennes; le $F$. Léandre Dugal qui a fondé, il y a 25 ans, le Service de préparation à la vie (SPV) qui s'inspire des premières communautés chrétiennes pour éduquer à la foi les jeunes. Ces deux mouvements rejoignent actuellement des milliers de jeunes dans plus d'une trentaine de diocèses. Ils ont rejoint les jeunes d'ici en paroisse et dans les écoles.

Si vous voulez en savoir davantage sur les origines, vous pourrez lire du F. Antoine Bernard, L'Histoire des Clercs de Saint-Viateur au Canada et la Vie du P. Étienne Champagneur. Et du P. Léo-Paul Hébert, Le Québec de 1850 en lettres détachées, récemment édité par le ministère des Affaires culturelles du Québec. Il nous donne, à travers la correspondance des premiers Clercs de Saint-Viateurs avec les confrères de France, leur vision de la vie québécoise de cette époque.

Et si vous voulez savoir comment la communauté a relevé les défits de la société contemporaire, vous pourrez lire du F. Paul-André Turcotte, ses volumes parus chez Bellarmin: L'éclatement d' un monde (Les Clercs de Saint-Viateur et la Révolution tranquille); Les chemins de la différence (Pluralisme et aggiornamento dans l'après-Concile).

Fidèles, non pas nécessairement au passé, mais au souffle inspirateur de Querbes, c'est avec audace et détermination que la communauté entrevoit l'avenir en tentant, comme son fondateur, de répondre aux exigences du monde présent, là où l'Église l'envoie aujourd'hui à travers le monde!

Actuellement, la grande famille de Querbes compte plus de 1000 religieux qui travaillent dans 15 pays. La maison générale est à Rome. Cette année encore, comme l'an dernier, une trentaine de jeunes se préparent dans les noviciats de la communauté à assurer la relève. 